\title{
Low Complexity Frequency Domain Equalization of MIMO Channels with Applications to MIMO-CDMA Systems
}

\author{
A. Burg \\ IIS,ETH-Zurich \\ apburg@iis.ee.ethz.ch
}

\author{
M. Rupp \\ TU-Wien \\ mrupp@nt.tuwien.ac.at
}

\author{
S. Haene, D. Perels, N. Felber, W. Fichtner \\ IIS,ETH-Zurich \\ haene,perels,felber,fw@iis.ee.ethz.ch
}

\begin{abstract}
CDMA and MIMO-CDMA systems using RAKE receivers are heavily limited by self- and multiple-accessinterference. Linear equalization is a means to remove this interference, however it is often not practical due to the enormous complexity, especially in the MIMO case. This paper presents an approach to greatly reduce the complexity of linear MIMO equalizers. It discusses the complexity reduction of the equalizer itself and describes a suboptimal low-complexity method to compute its coefficients. The application of frequency domain equalization using the overlap/add FFT method to MIMO systems is suggested. The coefficients of the joint equalizer/MIMO detector are also derived in the frequency domain, based on an approximation of an MMSE criterion. Performance results in terms of BER are quantified through simulations of a MIMO-extended UMTS-FDD downlink.
\end{abstract}

\section{INTRODUCTION}

Spatial multiplexing has been suggested as a means to greatly increase the capacity of wireless communication systems [1]. After it was initially applied to narrow-band systems it has recently been suggested for OFDM and CDMA. In OFDMbased systems, a cyclic prefix avoids interference from multipath propagation and allows straight-forward equalization in the frequency domain (FD). If properly exploited the multipath propagation adds additional diversity and is therefore an overall advantage. In single-carrier CDMA systems the diversity gain is often negligible compared with the resulting interference, especially in a heavily loaded system. This also holds in the case of MIMO-extended CDMA systems [2]. Among numerous other methods [3], linear equalization has been suggested as a technique to remove this interference. However, it is noted that its actual straight-forward implementation is often not feasible or not economic due to the enormous complexity of the equalizer itself and of the computation of its coefficients. This paper proposes possible solutions to both problems and is organized as follows: The next section discusses the application of the MIMO-equalizer to the received data stream in the frequency domain and quantifies the resulting complexity reduction. It is argued that in the MIMO case FD equalization becomes very efficient even for a small number of taps. The problem of obtaining the equalizer coefficients is addressed in section three. Section four compares the performance of the FD solution with a standard time domain (TD) MMSE solution. The paper is concluded in section five.

\section{EQUALIZER IMPLEMENTATION}

The general structure of a linear MIMO equalizer is shown in figure 1 for a $2 \times 2$ configuration. It has been shown that this approach can successfully remove interference from multipath propagation [3], [4]. However, the paramount complexity of the matrix convolution often prohibits its application except for very short channels.

\section{A. Algorithm}

Various, in principal similar approaches have been suggested to reduce the complexity of SISO FIR filters through their realization in the frequency domain or by using oversampled filter banks [5]. They all split the received signal into a number of subbands either through an FFT or through analysis filters. All subbands are subsampled and processed individually at a lower rate before they are recombined using an IFFT or a set of appropriate synthesis filters. These techniques avoid or shorten the costly convolution operation. The same approach can also be applied to linear MIMO equalizers.

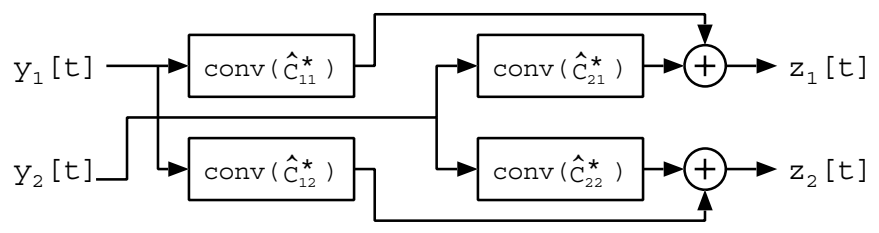

Fig. 1. Linear 2x2 TD-MIMO equalizer

In this paper the overlap/add method is applied to a MIMO system with $N_{t x}$ transmit and $N_{r x}$ receive antennas. A block diagram of the algorithm is shown in figure 2 for the $2 \times 2$ case. A sequence of $L_{e}$ samples of each received signal $y_{n}[t]$ $\left(n=1, \ldots, N_{r x}\right)$ is collected first using serial to parallel conversion to form the vectors $\mathbf{y}_{n}$. At the same time $L_{e}$ defines the length of the equalizer, which for efficiency of the FFT is chosen to be a power of 2 . Next, all $\mathbf{y}_{n}$ are zero-extended to a length of $L \geq L_{c}+L_{e}-1$, with $L_{c}$ denoting the length of the channel impulse response (CIR). For efficiency this value is chosen to be $L=2 L_{e}$, which is valid since $L_{e} \geq L_{c}-1$. The results are transformed into the frequency domain. There, they are multiplied component-wise with the oversampled frequency domain equivalents of the $N_{t x} N_{r x}$ partial MIMO equal- 
izer impulse responses. The corresponding results from the different channels are then added appropriately, resulting in $N_{t x}$ streams which are transformed back into the time-domain. The frequency-domain separation of the spatially multiplexed components resembles thereby the MIMO detection in an OFDM system. However, due to the absence of a cyclic prefix a linear convolution is required. The overlap/add procedure performs this by delaying the second half of the resulting vectors by one block and adding the first half to the retained subblock from the preceding iteration (see [5] and references therein).

\section{B. Complexity}

To analyze the complexity of the application of the equalizer coefficients it is necessary to distinguish between the order of a scheme which is an asymptotic measure and the actually required number of operations. It is well known that FDequalization reduces the complexity (per sample) from order $O\left(L_{e}\right)$ to $O\left(\log \left(L_{e}\right)\right)$ making it the more efficient choice as $L_{e}$ becomes large. However in practice, in terms of number of operations, a certain minimal length is required before the reduced order leads to lower computational requirements. This fact is illustrated in figure 3 which plots the approximate (upper bound) number of complex multiplications versus the number of filter taps. The corresponding equations in Table I are derived based on an unoptimized, straight forward radix-2 FFT implementation. The use of a radix-4 FFT can reduce overhead complexity further. In addition to that, pruning of operations on constant zero samples also has the potential of saving up to $L_{e}$ complex multiplications per FFT. It can be seen that, as the number of antennas becomes larger, the FD implementation becomes more efficient than the TD-implementation even for fewer coefficients. The reason for this is the fact that only $N_{r x}+N_{t x}$ FFTs/IFFTs are required for a system with otherwise $N_{t x} N_{r x}$ filters. The MIMO case therefore provides proportionally higher savings in terms of operations per sample than the SISO case, since the relative overhead from the FFT and overlap/add operation is reduced.

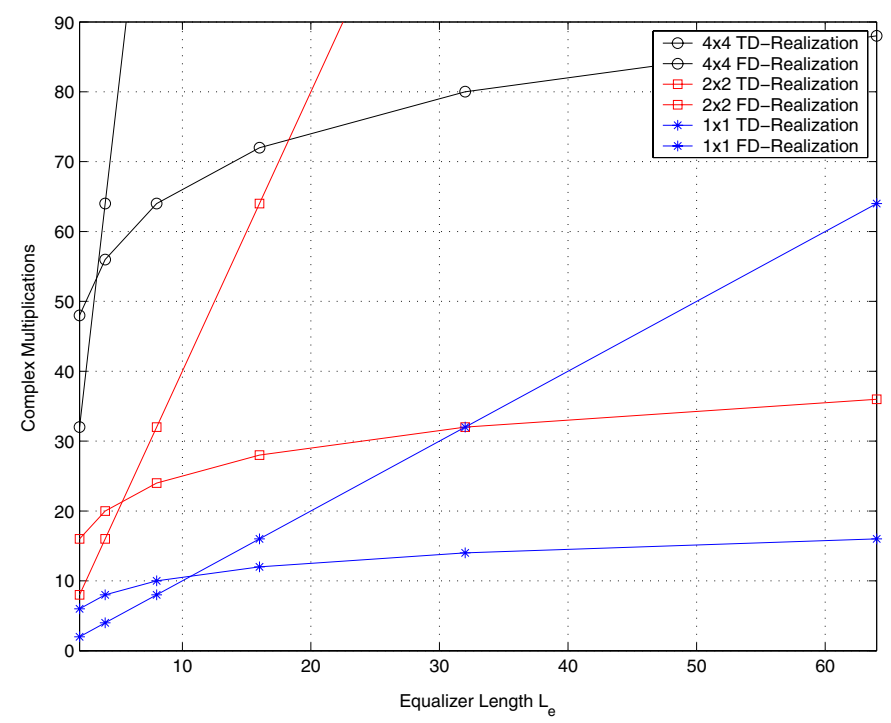

Fig. 3. Complexity of the frequency domain MIMO equalizer
TABLE I

EQUALIZER COMPLEXITY IN COMPLEX MULTIPLICATIONS PER SAMPLE

\begin{tabular}{|c|c|c|}
\hline & $\begin{array}{c}\text { Complex multiplications } \\
\text { per sample }\end{array}$ & Complexity \\
\hline Time Domain & $N_{t x} N_{r x} L_{e}$ & $O\left(L_{e}\right)$ \\
\hline Freq. Domain & $\begin{array}{c}\left(N_{t x}+N_{r x}\right) \log _{2}\left(2 L_{e}\right) \\
+2 N_{r x} N_{t x}\end{array}$ & $O\left(\log \left(L_{e}\right)\right)$ \\
\hline
\end{tabular}

\section{Computation of the Equalizer Coefficients}

Efficiently computing the MIMO equalizer coefficients is another challenge. A variety of iterative methods which exhibit a reasonable complexity and can achieve close-to-optimal steady state performance exists for the SISO case and [6], [7], [8] have recently applied them to the MIMO case. However, they all suffer from the fact that they require time to converge and are consequently not well applicable under fast changing channel conditions. In this paper an approach similar to the so called window method for the design of FIR filters is proposed. The algorithm is first explained for the SISO case and is later extended to the MIMO case.

\section{A. SISO Case}

The starting point is the infinite-length sampled channel impulse response $h[t]$. Using the Fourier-transform operators $\mathfrak{F}$ and the inverse $\mathfrak{F}^{-1}$, the exact infinite-length equalizer solution can be obtained in the frequency domain as

$$
c[t]=\mathfrak{F}^{-1}\left\{\frac{\mathfrak{F}\{\delta[t-D]\}}{\mathfrak{F}\{h[t]\}}\right\} .
$$

It is now assumed that the CIR is of finite length $L_{c}$ $\left(h\left[t \geq L_{c}\right]=0, h[t<0]=0\right)$ and that the length of a practical equalizer is given by $L_{e} \geq 2 L_{c}$. In this case (similar to the overlap/add method) the linear convolution between the equalizer and the CIR can be expressed as a circular convolution if $h[t]$ and $c[t]$ are both zero extended to length $L \geq L_{e}+L_{c}-1$ and are periodically repeated to form $\hat{h}[t]$ and $\hat{c}[t]$. For practical reasons $L$ is chosen to be $L=2 L_{e}$ which will always satisfy the previous constraint. Unfortunately, the equalizer solution can not be computed directly by substituting $\mathfrak{F}$ with DFT in equation 1 , since the resulting impulse response would also be of length $2 L_{e}$. This would violate the vital constraint that was imposed to allow the transition from the linear to a circular convolution. Instead, each period of $\hat{c}[t]$ is rewritten as $\hat{c}[t]=\widetilde{c}[t] w[t]$, whereby $\widetilde{c}[t]$ and the window function $w[t]$ are periodic with $2 L_{e}$ and are fully described by :

$$
\begin{gathered}
\widetilde{c}[t]=\left\{\begin{array}{cc}
c[t] & , 0<t<L_{e} \\
c\left[t-L_{e}\right] & L_{e} \leq t<2 L_{e}
\end{array}\right. \text { and } \\
w[t]=\left\{\begin{array}{cc}
1 & , 0<t<L_{e} \\
0 & L_{e} \leq t<2 L_{e}
\end{array}\right.
\end{gathered}
$$

Herewith, the frequency domain solution can be formulated based on the discrete Fourier transform (DFT):

$(\operatorname{DFT}\{\widetilde{c}[t]\} \star \operatorname{DFT}\{w[t]\}) \operatorname{DFT}\{\hat{h}[t]\}=\operatorname{DFT}\{\delta[t-D]\}$ 


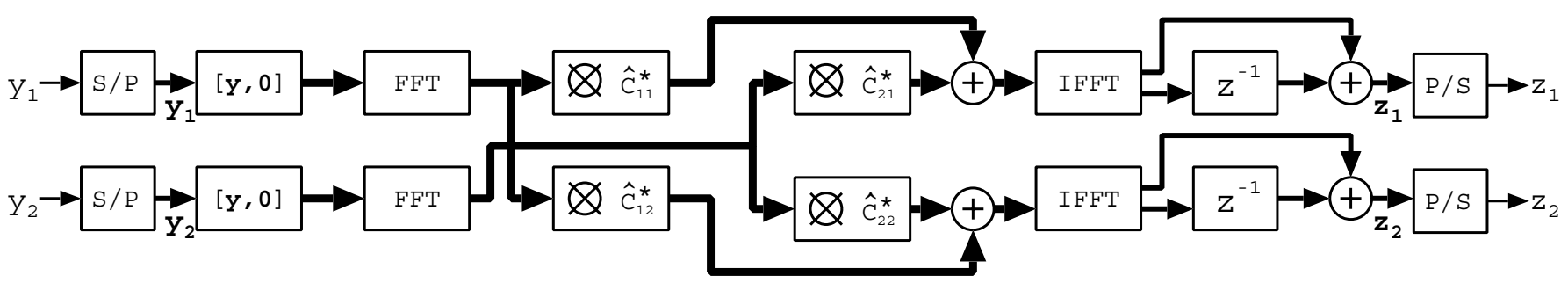

Fig. 2. 2x2 MIMO equalizer using the overlap/add method

Unfortunately, as a consequence of the convolution with the FD representation of the window function, the equations for the coefficients are no longer decoupled and virtually no advantage in terms of complexity would be gained from the FD approach.

To circumvent this problem an approximation $\tilde{w}[t]$ to $w[t]$ needs to be chosen which yields a more convenient (decoupled) expression from equation 3 . Thereto, it is first noted that all odd coefficients of DFT $\{\widetilde{c}[t]\}$ over the length of $2 L_{e}$ are zero. as a This is a consequence of $\widetilde{c}[t]$ having a period of length $L_{e}$. Independence of the equations for the $L_{e}$ free coefficients is now guaranteed if the frequency domain representation of the new window is chosen in such a way that only its first two coefficients are non-zero (DFT $\{\tilde{w}\}[f>1]=0)$. At the same time, its TD representation should be the best possible match for the original rectangular window $w[t]$. The following appears to be a suitable choice:

$$
\tilde{W}[f]=\operatorname{DFT}\{\tilde{w}[t]\}=\left\{\begin{array}{cl}
1 & , f=0 \\
\exp \left(-j \frac{2 \pi}{4}\right) & , f=1 \\
0 & , 1<f<2 L_{e}
\end{array} .\right.
$$

Its TD and FD representation are depicted in figure 4 together with the original window function.
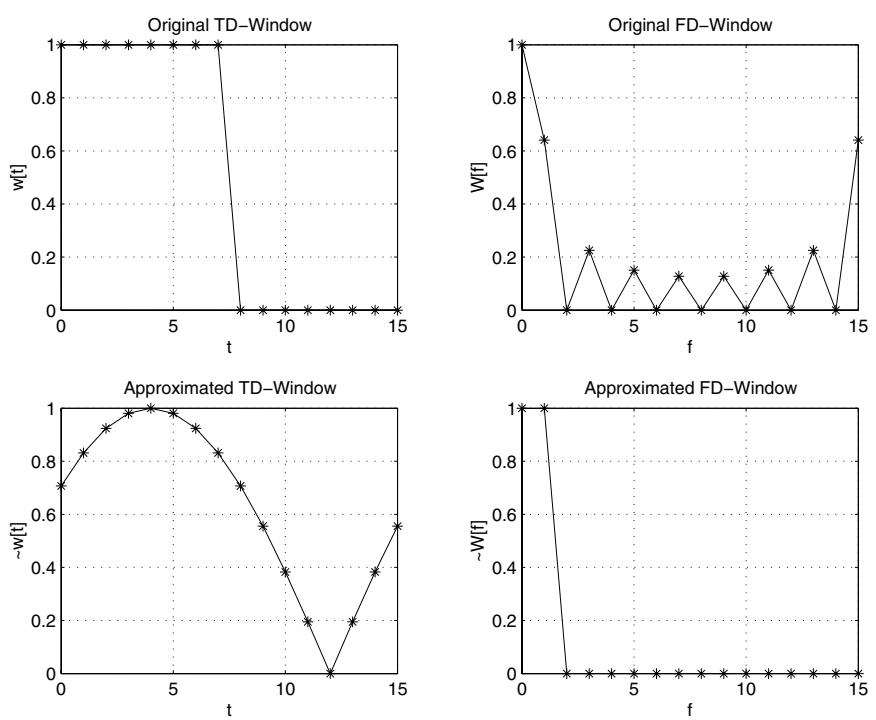

Fig. 4. TD and FD representations of the original $(w[t])$ and approximated $(\tilde{w}[t])$ windows for $L_{e}=8$

With this approximation a set of $L_{e}$ mutually decoupled pairs of equations is obtained from equation 3. Each of them can be written as follows:

$$
\begin{aligned}
C[f] \tilde{W}[0] \hat{H}[2 f] & =T[2 f] \\
C[f] \tilde{W}[1] \hat{H}[2 f+1] & =T[2 f+1]
\end{aligned}
$$

where $C[f], \hat{H}[f]$ and $T[f]$ are the FD representations of the impulse responses of $c[t], \hat{h}[t]$ and of the target impulse response $\delta[t-D]$ respectively. To determine the minimumnorm solution, the equations in 5 are rearranged in matrix notation as $\hat{\mathbf{Q}}[f]=[\tilde{W}[0] \hat{H}[2 f] \quad \tilde{W}[1] \hat{H}[2 f+1]]^{T}$ and $\mathbf{T}[f]=\left[\begin{array}{ll}T[2 f] & T[2 f+1]\end{array}\right]^{T}$. This finally leads to the following expressions for the FD representation of the zeroforcing and the MMSE equalizer solutions for a known signalto-noise ratio $\rho$ :

$$
\begin{gathered}
C_{Z F}[f]=\left(\hat{\mathbf{Q}}[f]^{H} \mathbf{T}[f]\right) /\left(\hat{\mathbf{Q}}[f]^{H} \hat{\mathbf{Q}}[f]\right) \\
C_{M M S E}[f]=\left(\hat{\mathbf{Q}}[f]^{H} \mathbf{T}[f]\right) /\left(\hat{\mathbf{Q}}[f]^{H} \hat{\mathbf{Q}}[f]+\frac{N_{t x}}{\rho}\right)
\end{gathered}
$$

The so obtained coefficients can now be transformed back into the time domain: $\mathbf{c}=\operatorname{IDFT}\{\mathbf{C}\}$. However, if the equalizer is implemented based on the overlap/add or overlap/save method as suggested previously, the FD coefficients $C[f]$ can not be used directly, since an oversampled spectrum is required. This can either be obtained by first finding the TD solution, zeroextending it to a length of $2 L_{e}$ and transforming it back to FD (see figure 5a). Alternatively, the upsampling can be performed directly in the FD as shown in figure 5b. If only an approximation is required the convolution consists of only very few coefficients and the second alternative tends to be more efficient.

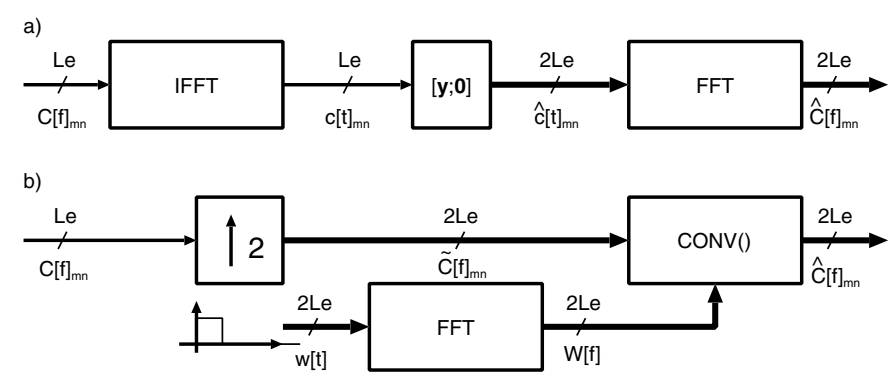

Fig. 5. Upsampling of the equalizer spectrum for overlap/add method

\section{B. Extension to the MIMO case}

The extension of this algorithm to a MIMO system is straight forward: A set of CIRs $\mathbf{h}^{p, q}$ exists describing the propa- 
gation from transmit antenna $q$ to the receiving antenna $p$. The zero extension and transformation into the frequency domain can be performed on all of them independently. The elements of the FD-MIMO channel $\hat{H}[f]$ now become matrices $\hat{\mathbf{H}}[f]$ which fully describe the MIMO channel at the individual frequency components. The desired result $\mathbf{T}[f]$ in equation 6 also takes the form of a matrix $\mathbf{T}[f]=$ $\left[\begin{array}{cc}T[2 f] \mathbf{I}^{N_{t x} \times N_{t x}} & T[2 f+1] \mathbf{I}^{N_{t x} \times N_{t x}}\end{array}\right]^{T}$. Except for the oversampling this resembles the process required to find the equalizer solution and MMSE antenna separation for a MIMOOFDM system. The transformation back to the TD or the oversampling for the overlap/add method is again performed on all $N_{t x} N_{r x}$ channels independently and analogous to the SISO case.

\section{Complexity}

The FD solution exhibits a significantly lower complexity than the full TD-MMSE solution described in [3]. The described (suboptimal) approach requires only $L_{e}$ matrix inversions of dimension $N_{t x} \times N_{t x}$, while the full exact solution would require a single matrix inverse of dimension $L_{e} N_{t x} \times$ $L_{e} N_{t x}$. Noting that the complexity of this operation is of order $O\left(n^{3}\right)$, it is evident that the FD-algorithm is much more desirable. An exact quantification in terms of number of operations is fairly difficult as it depends strongly on the implementation of the matrix-inversion. In addition to that it is important to note that the direct method also requires the implementation of a channel estimator which, for example, is not needed if an adaptive LMS method is used.

\section{Simulation Results}

To assess the performance of the above proposed FD-MMSE algorithm it is compared to the full TD-MMSE solution, which is also reported in [4]. Simulations are based on a 4x4 MIMOextended downlink, as described in [9]. A spreading factor of 32 was used and the length of the equalizer was set to $L_{e}=32$. Perfect knowledge of the static Rayleigh channel and the SNR (defined as the ratio between the power of a single user and the AWGN before despreading) was assumed at the receiver. In a first experiment the number of concurrent users was set to $K=1,8,16$ and the per-user SNR (proportional to the $E_{b} / N_{o}$ in the system) was varied from $-14 \mathrm{~dB}$ to $+14 \mathrm{~dB}$. The results are shown in figure 6 for a channel with three paths of equal power spaced two chips apart and in figure 7 for a channel with a VehicularA power delay-profile. In both cases the FD-MMSE solution follows closely the TD solution for the case where only one user is active. With 8 concurrent users in the system the FD-MMSE solution initially keeps up with the TD solution, however in the high-SNR regime it appears to have more residual interference which leads to some performance degradation compared to the TD solution and eventually to an error floor. With 16 active users the BER curves of the FD-MMSE equalizer level off at an error floor of $10^{-3}$ for the channel with three equal taps and at $2 \cdot 10^{-4}$ for the VehicularA channel respectively. For comparison the results for a space-time (ST) RAKE receiver are also shown. Especially for a higher number of users it is evident that both equalizer solutions outperform the RAKE receiver significantly.

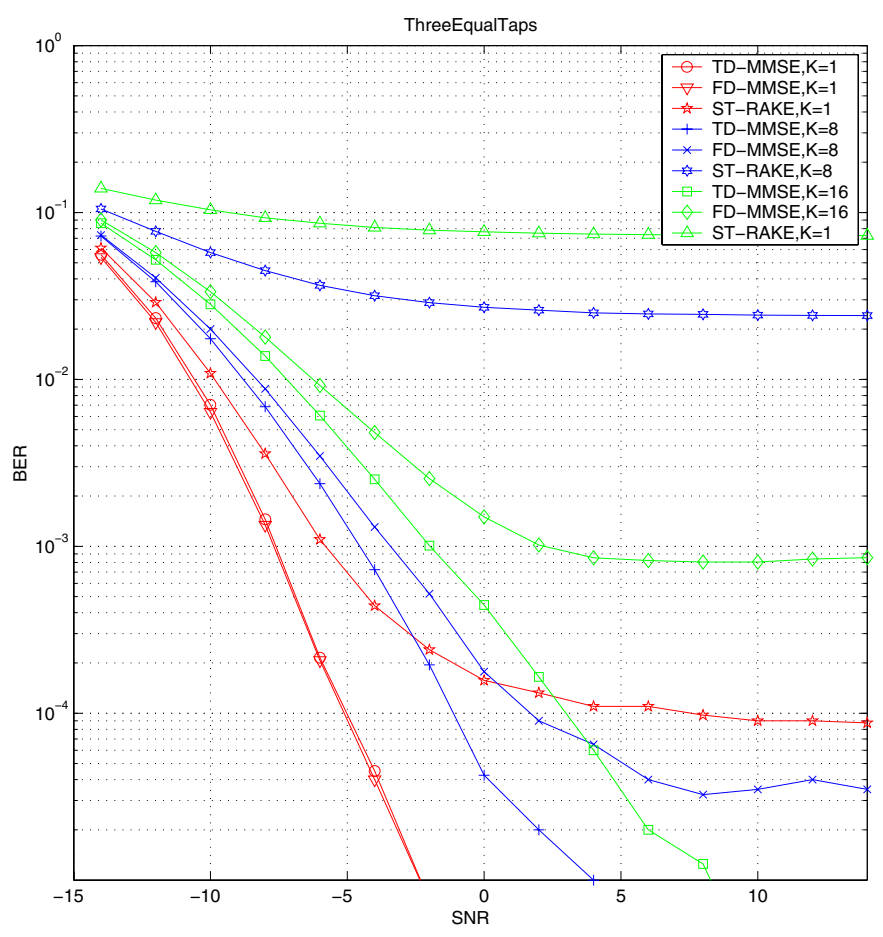

Fig. 6. BER v.s. per user SNR for TD/FD-MMSE linear equalizer and STRAKE receiver through a channel with three taps of equal power

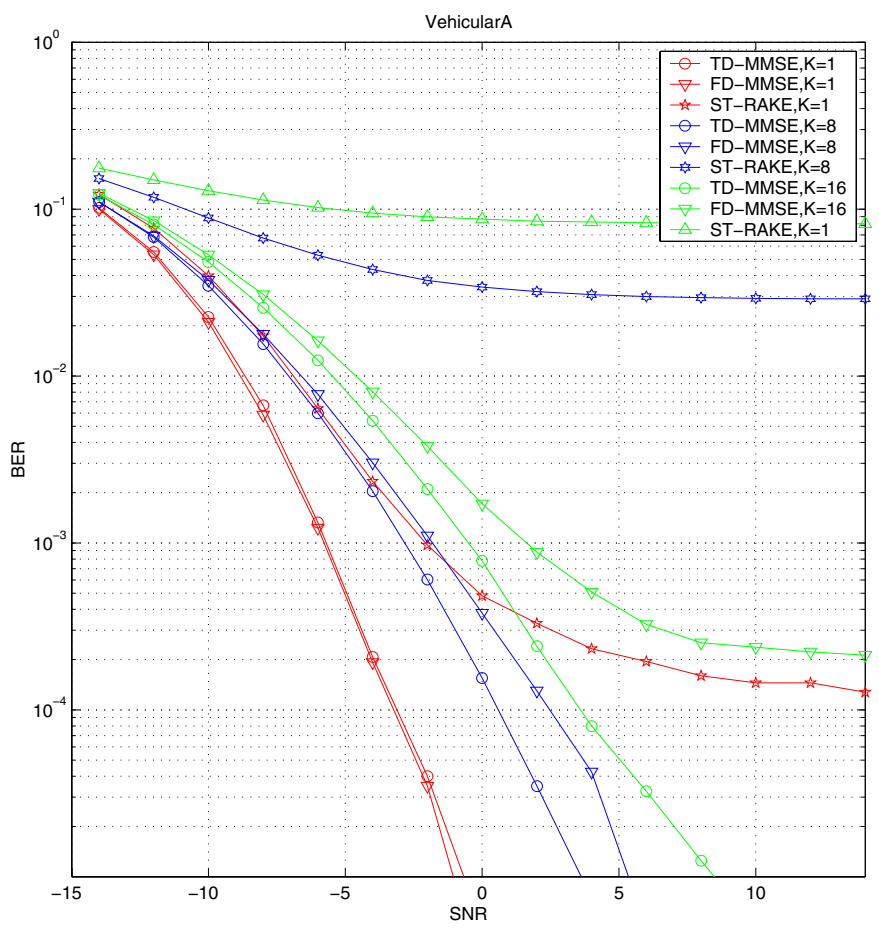

Fig. 7. BER v.s. per user SNR for TD/FD-MMSE linear equalizer and STRAKE receiver through a VehicularA channel

A different view is taken in figure 8. It assumes a constant $E_{b} / N_{o}$ or equivalently a constant per user SNR while showing 
the BER performance for an increasingly loaded system in a $1 \times$ $1,2 \times 2$ and $4 \times 4$ configuration. To compensate for the increased spatial rate and to maintain a constant data rate the spreading factor is increased proportionally to the number of antennas. Again, the gap between both equalizer solutions and the STRAKE receiver becomes evident. With a growing number of users the BER of the system using the FD equalizer is observed to increase faster than with the TD MMSE equalizer. This is due to the higher mean-square error in the FD approximation. However, the proposed FD MMSE solution still provides an interesting tradeoff between complexity and performance.
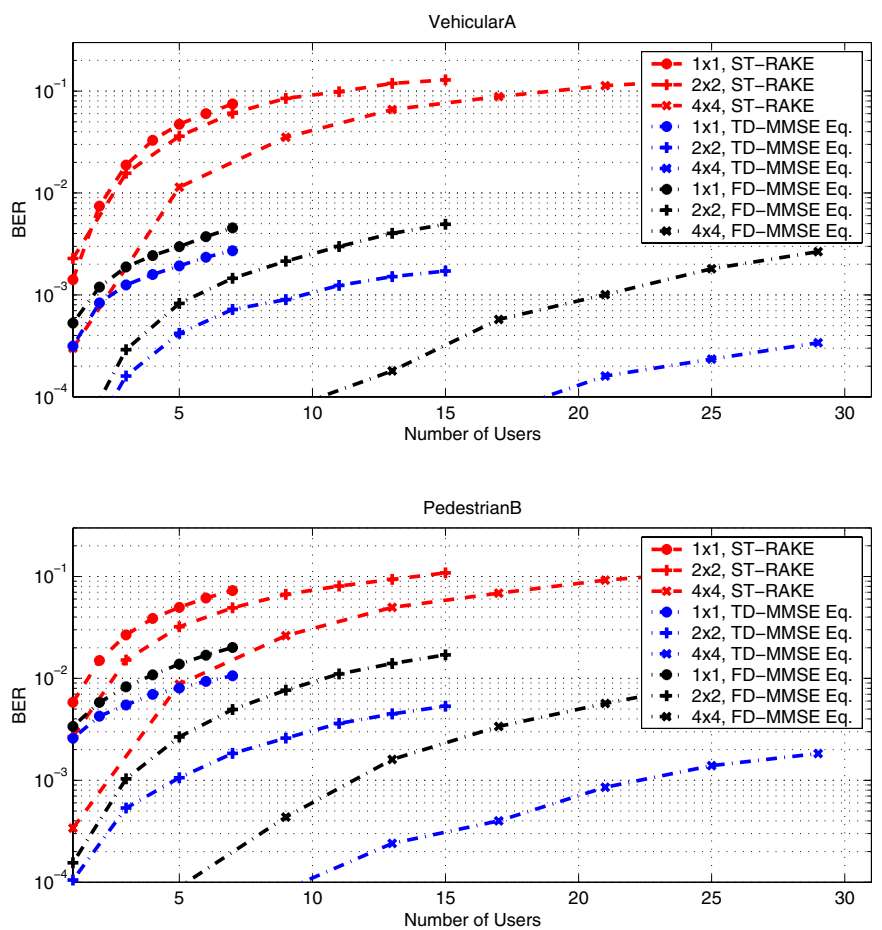

Fig. 8. BER v.s. number of users for TD/FD-MMSE and ST-RAKE receiver for VehicularA and PedestrianB channels at a constant $E_{b} / N_{o}$

\section{CONCLUSION}

In this paper low complexity linear MIMO equalization is discussed. The application of the overlap/add method is suggested to greatly reduce the complexity of the MIMO equalizer itself without any loss in performance. The achievable gain in terms of number of significant operations (complex multiplications) is quantified. Furthermore, an algorithm to efficiently compute the equalizer coefficients in the frequency domain is devised. While it can not achieve the full performance of the straight-forward TD MMSE solution, it is shown to still provide excellent interference suppression while only requiring a fraction of the original number of operations.

\section{REFERENCES}

[1] G. J. Foschini, "Layered Space-Time Architecture for Wireless Communications in a Fading Environment When Using Multi-Element Antennas", Bell Labs Technical Journal, Vol. 1, No. 2, pp.315-335, Autumn 1996

[2] Samardzija, D.; Wolniansky, P.; Ling, J.: "Performance evaluation of the VBLAST algorithm in W-CDMA systems", in Proc. IEEE VTC-Fall, 2001, Vol. 2, pp. $723-727$
[3] B. A. Bjerke, J. G. Proakis: "Equalization and Decoding for Multiple-Input Multiple-Output Wireless Channels", EURASIP Journal on Applied Signal Processing, Vol.3, pp. 249-266, March 2002

[4] A. Burg, M. Rupp, D. Perels, N. Felber, W. Fichtner: "Performance of MIMO-extended UMTS-FDD Downlink using Space-Time RAKE and Linear Equalizer", in Proc. IEEE VTC-Fall, 2003

[5] J.J. Shynk: "Frequency-Domain and Multirate Adaptive Filtering", IEEE Signal Processing Magazine, Vol. 9, pp. 14-37, Jan. 1992

[6] A. Maleki-Tehrani, B. Hassibi, J.M. Cioffi: "Adaptive Equalization of Multiple-Input Multiple-Output (MIMO) Frequency Selective Channels", in Proc. IEEE ICC, 2000, Vol.3, pp. 1670-1674

[7] L. Mailaender, "Linear MIMO Chip Equalization for the CDMA Downlink", in Proc. IEEE SPAWC, 2003

[8] G. Woodward, L. Mailaender, S. Venkatesan, "Chip Equalization for Third Generation MIMO High Data Rate Applications", in Proc. IEEE Workshop on Sensor Array Multichannel Signal Processing, 2002

[9] A. Adjoudani, E. Beck, A. Burg, G. Djuknic, T. Gvoth, D. Haessig, S. Manji, M. Milbrodt, M. Rupp, D. Samardzija, A. Siegel, T. Sizer II, C. Tran, S. Walker, S. A. Wilkus, P. Wolniansky: "Prototype Experience for MIMO BLAST over Third Generation Wireless System", IEEE JSAC, Vol. 21, No. 3, pp. 440-451, April 2003 